\title{
RADIOACTIVE POLLUTION GENERATED BY THE ELECTRIC POWERS USING COAL AS COMBUSTIBLE
}

\author{
Petru MURSA, Florin VOCHECI, Răzvan-Olimpiu DUMITRESCU, \\ Laurenţiu Daniel Nicolae ŞUFARU
}

\author{
Scientific Research Center for CBRN Defence and Ecology, Bucharest, Romania \\ petrumursa73@gmail.com, florinvocheci@yahoo.com, \\ olimpiu81@yahoo.com, laurentiu16@lycos.com
}

\begin{abstract}
A type of NORM (Normally Occurring Radioactive Materials) category is coal used as a fuel in power plants. The ash resulting from their burning is stored in the heaps near the thermal power stations and has a multiplication factor of the radionuclide concentration between 2 and 10, compared to the coal from which it originates. Even if in this case the radioactive pollution does not represent a significant danger for the population, this ash can be used in the construction of building materials, and thus through radon disintegration products emanating from these materials, can contribute to increasing the dose of ionizing radiation of the population, knowing that most of their lives and spend it inside buildings.
\end{abstract}

Keywords: thermal power, coal, radioactive ash, radon

\section{Introduction}

The extraction and use of non-ferrous and radioactive metals, coal, geothermal waters, the operation of atomic power plants and nuclear explosions during the 1950s and 1960 s altered the concept of radioactivity of the environment and led to the need for control and surveillance in all the components that define the notion of environment $[1,2]$. Thus, the radioactivity of the environment is given, together with the natural radionuclides existing since the formation of the Earth, and radioactive substances created exclusively in human activities (civil and military industries, medicine, scientific research, energetic reactors, waste etc.). Natural radioactivity is given by the cosmic radiation and the radioactive elements contained in the Earth's crust since the formation of our planet. Approximately 340 nuclides are found in the environment, of which 70 are radioactive $[3,4]$. Earth's natural radioactivity includes three major components:

a. Primordial radionuclides, having halflives long enough to survive since they were created (those who are the heads of the three natural radioactive series);

b. Progenitors of these primordial radionuclides;

c. Cosmogenic radionuclides formed by the interaction of cosmic radiation with the atmosphere [5].

The activity of naturally occurring radionuclides and naturally occuring radioactive materials (NORM) can be artificially intensified by various technological processes, resulting in a new category of radionuclides called Technologically Enhanced Naturally Occurring Radiative Materials (TENORM) 
[6]. Lately, there has been an increase in the awareness of the danger of natural radioactive materials (NORM) as well as the increase in their concentrations in various non-nuclear industrial processes. There are cases where these naturally enriched radioactive materials (TENORM) may have the same levels of activity as waste with low levels of radioactivity from the nuclear fuel cycle. [7]

Artificial radioactivity is the result of experiments with nuclear weapons, the use of nuclear power plants and the use of manmade radionuclides in various applications (medicine, scientific research, industry, etc.) over 120 years after the discovery of radioactivity by Henry Bequerel.

In conclusion, the radioactivity of the environment (soil, air, water, food, plants, etc.) comes from natural radioactivity, but also from artificial radioactivity.

\section{Chemical, radioactive and noise pollution generated by thermal power plants}

As we know, technology, along with the extraordinary advantages offered to mankind, has brought many shortcomings, of which an important place is the pollution of the environment.

A thermoelectric or thermal power plant is an electric power plant that generates electricity based on the transformation of the thermal energy resulting from combustion of fuels. The electric current is produced by electric generators driven, in most cases, by steam turbines.

Materials resulting from coal combustion in thermo-power stations are:

- clay deposited on the bottom of the oven; is "heavy ash" and is the mineral part of coal; represents $20 \%$ of the combustion material;

- ash or "light ash" or "flying ash" that escapes from the chimney filters and reaches the atmosphere and then deposits on the ground; represents $80 \%$ of the combustion material;
- radon released into the atmosphere, increasing radioactivity even through its disintegration products, $\mathrm{Bi}-214$ and $\mathrm{Pb}-210$; - hot gases.

In the burning process, harmful substances such as sulfur oxides (Sox), nitrogen oxides (NOx), carbon oxides (Cox) and ash dust are generated. Along with these, small amounts of hydrochloric and hydrofluoric acid are also produced.

Fly ash discharged through the chimneys of combustion plants of thermal power plants together with fine ash dust from ash storage dumps and coal dust from coal dumps or from transport or preparation is a solid noxious matter in the form of aerosols. [8] In addition, the installations and equipment used for the coal supply of steam boilers, namely the sorting-crushing plant, the conveyor belts and the cup-drive machines, produce continuous noise pollution.

The dispersion of industrial, chemical and radioactive pollutants depends on the following factors:

- factors that characterize the emission source (exhaust sump height, its diameter, gas velocity and temperature, the amount of pollutant emitted per unit time, the physical and chemical properties of the pollutant);

- factors that characterize the area where the emission took place (topography and roughness of the land);

- meteorological factors (air currents characterized by direction and velocity, thermal stratification of the atmosphere).

Dispersing in the environment, pollutants of different types get into the air, water, soil [9-19] and produce numerous negative effects on plants [20-27], animals, and humans [28].

\section{Radiation pollution generated by thermal power plants}

Average annual ash and slag yields from a coal-fired power plant vary mainly with the installed power of the power plant, the quality and degree of coal fragmentation, and the characteristics of the combustion boiler. 
In our country, at all power stations that use coal as the main fuel, the transport of ash to the dumps is made by hydraulic systems because of the large quantities to be transported.

In Romania the ash dumps do not have high heights, but occupy a large area of land.

Ash and slag represent the unaltered solid residues resulting from the combustion of coal in power plants and consist of calcite, pyrite, clay and other minerals that are divided and decomposed in the combustion process.

The main fraction resulting from combustion is ash $(70 \div 90 \%)$ compared to slag and other resulting materials. Its color may be gray or light gray, depending on the unbound carbon content.

All coal varieties contain natural radioactive isotopes in certain concentrations. By burning them in the center, there is a concentration of radioactive isotopes in the combustion products which are subsequently discharged into the environment. [29]

The table below shows the specific radioactive activities of the main radionuclides contained in the coal extracted from the mines in Romania:

Table 1 The specific radioactive activities of the main radionuclides contained in the coal extracted from the mines in Romania

\begin{tabular}{|c|c|}
\hline $\begin{array}{c}\text { Natural } \\
\text { radioisotope }\end{array}$ & $\begin{array}{c}\Lambda_{\mathrm{s}} \\
(\mathrm{Bq} / \mathrm{kg})\end{array}$ \\
\hline${ }^{40} \mathrm{~K}$ & $30 \div 650$ \\
\hline${ }^{238} \mathrm{U}$ & $1 \div 100$ \\
\hline${ }^{232} \mathrm{U}$ & $1 \div 50$ \\
\hline${ }^{226} \mathrm{Ra}$ & $1 \div 150$ \\
\hline
\end{tabular}

Charcoal contains ${ }^{40} \mathrm{~K}$, uranium, thorium The specific activity of the main and all radionuclides in the disintegration chains. Also, radon from the natural radionuclide disintegration chain is exalted by dislocation and granulation inside the combustion chamber, increasing the concentration of radon within it.

Table 2 The specific activity of the main radionuclides in the fly ash emitted from radionuclides in the fly ash emitted from coal-fired power plants in Romania is presented in the table below:

\begin{tabular}{|c|c|}
\hline $\begin{array}{c}\text { Natural } \\
\text { radioisotope }\end{array}$ & $\begin{array}{c}\Lambda_{\mathrm{s}} \\
(\mathrm{Bq} / \mathrm{kg})\end{array}$ \\
\hline${ }^{40} \mathrm{~K}$ & $160 \div 1200$ \\
\hline${ }^{238} \mathrm{U}$ & $2 \div 312$ \\
\hline${ }^{226} \mathrm{Ra}$ & $3 \div 520$ \\
\hline${ }^{210} \mathrm{~Pb}$ & $10 \div 500$ \\
\hline${ }^{210} \mathrm{Po}$ & $2 \div 170$ \\
\hline${ }^{232} \mathrm{Th}$ & $2 \div 170$ \\
\hline
\end{tabular}

It can be seen that the radioactivity resulting from the combustion of coal is 2 $\div 10$ times higher than that of the primary fuel.

Worldwide, according to the literature, the average specific activity of $238 \mathrm{U}$ and $232 \mathrm{Th}$ of coal is about $20 \mathrm{~Bq} / \mathrm{kg}$, with values ranging from 5 to $300 \mathrm{~Bq} / \mathrm{kg}$.
During coal combustion, organic compounds are gasified (water vapor and carbon dioxide), and inorganic ones containing naturally occurring radionuclides are concentrated in ash. Generally, the radionuclide amplification factor in ash, as shown in the tab. no. 2 is approximately equal to 10. Taking into account the low 
migration rate of ash radioisotopes, the use of this material in road construction is not subject to major restrictions.

However, the use of ash as building material for buildings and dwellings may result in direct and indirect exposure of the population through radon emanations, the main routes of exposure being the ingestion and inhalation of radioisotopes $\mathrm{Pb}-210$ and Po-210. [30]

The table below lists the values of the specific activities for ash samples from thermal power stations worldwide, values specified in the references in the first column of the table

Table 3 The specific activity of ashes from thermal power plants reported by foreign authors

\begin{tabular}{|c|c|c|c|c|c|c|c|c|c|}
\hline Reference & Sample & \multicolumn{8}{|c|}{ Radionuclide $(\mathrm{Bq} / \mathrm{kg})$} \\
\hline & & $\mathrm{Ra}^{226}$ & $\mathrm{Th}^{232}$ & $\mathrm{~Pb}^{210}$ & $\mathrm{U}^{238}$ & $\mathrm{U}^{235}$ & $\mathrm{~K}^{40}$ & $\mathrm{Po}^{210}$ & $\mathrm{Ac}^{228}$ \\
\hline $\begin{array}{l}\text { G.G. Pandit, S.K. Sahu and V.D. Puranik, } \\
\text { Natural radionuclides from coal fired thermal } \\
\text { power plants - estimation of atmospheric } \\
\text { release and inhalation risk, Radioprotection, vol. } \\
\text { 46, no. } 6 \text { (2011) S173-S179 EDP Sciences, } \\
2011\end{array}$ & 1 & 77,7 & $\begin{array}{c}125, \\
8\end{array}$ & 40,5 & 60,3 & - & - & - & - \\
\hline \multirow{10}{*}{$\begin{array}{l}\text { Craig Heidrich, Sue Brown, Doug Collier, } \\
\text { Naturally occurring radionuclides in Australian } \\
\text { coal combustion products, World of coal ash } \\
\text { Conference-Mai 9-12 Denver, CO, USA. }\end{array}$} & 1 & 110 & 120 & - & - & - & 450 & - & - \\
\hline & 2 & 90 & 130 & - & - & - & 190 & - & - \\
\hline & 3 & 110 & 150 & - & - & - & 570 & - & - \\
\hline & 4 & 30 & 70 & - & - & - & 100 & - & - \\
\hline & 5 & 60 & 90 & - & - & - & 320 & - & - \\
\hline & 6 & 140 & 200 & - & - & - & 210 & - & - \\
\hline & 7 & 7 & - & - & - & - & 20 & - & - \\
\hline & 8 & 40 & 7 & - & - & - & - & - & - \\
\hline & 9 & 160 & 280 & - & - & - & 70 & - & - \\
\hline & 10 & 150 & 290 & - & - & - & 60 & - & - \\
\hline $\begin{array}{l}\text { L. Mljač and M.Križman, Environmental } \\
\text { radioactivity due to fly-ash disposal results of a } \\
\text { monitoring programme, Symposium on } \\
\text { radiation protection in neighbouring countries in } \\
\text { Central Europe - } 1995\end{array}$ & 1 & 9,5 & - & - & 9,5 & - & - & - & - \\
\hline $\begin{array}{l}\text { Ts. Erkhembayar, T.Ulaanbaatar, M. } \\
\text { Baatarkhuu, N.Chimedtsogzol, Ts.Otgontuya, } \\
\text { Soil, Coal and Ash Radioactivity around } \\
\text { Baganuur Coal Deposit in Mongolia, Strategic }\end{array}$ & 1 & 163 & 39 & - & - & - & 196 & - & - \\
\hline $\begin{array}{l}\text { Technology (IFOST), 2013 8th International } \\
\text { Forum on } 03 \text { October } 2013\end{array}$ & 2 & 123 & 67,7 & - & - & - & 237 & - & - \\
\hline $\begin{array}{l}\text { Analysis of natural radionuclides in coal, slag } \\
\text { and ash in coal-fired power plants in Serbia - } \\
\text { M.M. Janković, D.J. Todorović, J.D. } \\
\text { Nikolić,University of Belgrade, Institute Vinča, } \\
\text { Radiation and Environmental Protection } \\
\text { Department, } 11001 \text { Belgrade, Serbia, } 2011\end{array}$ & 1 & 120 & 72 & - & 129 & 7,3 & 360 & - & - \\
\hline $\begin{array}{l}\text { J Suhana, M. Rashid, Air Resources Research } \\
\text { Laboratory, Malaysia - Naturally occurring } \\
\text { radionuclides in particulate emission from a } \\
\text { coal fired power plant: A potential } \\
\text { contamination ?, Journal of Environmental } \\
\text { Chemical Engineering, } 2016\end{array}$ & 1 & - & 13,2 & - & 9,8 & - & - & - & - \\
\hline & 1 & - & - & - & - & - & - & $\begin{array}{r}65,6 \\
\pm 6,7 \\
\end{array}$ & - \\
\hline & 2 & - & - & - & - & - & - & 136 & - \\
\hline
\end{tabular}




\begin{tabular}{|c|c|c|c|c|c|c|c|c|c|}
\hline \multirow{5}{*}{$\begin{array}{l}{ }^{210} \text { Po distribution after high temperature } \\
\text { processes in coal fired power plants - Wang } \\
\text { Chuangao, Liu Ruirui, Li Jinfeng, Huang } \\
\text { Zhijun, Pan Jingshun, Luo Zhiping, Chen Ling, } \\
\text { Wang Zhongwen, Pan Ziqiang, Department of } \\
\text { Radiation Safety, China, } 2017\end{array}$} & & & & & & & & \pm 32 & \\
\hline & 3 & - & - & - & - & - & - & $\begin{array}{c}181 \\
\pm 8,3\end{array}$ & - \\
\hline & 4 & - & - & - & - & - & - & $\begin{array}{c}117 \\
\pm 2,1\end{array}$ & - \\
\hline & 5 & - & - & - & - & - & - & $\begin{array}{l}94,1 \\
\pm 12 \\
\end{array}$ & - \\
\hline & 6 & - & - & - & - & - & - & $\begin{array}{c}64,5 \\
\pm 9 \\
\end{array}$ & - \\
\hline \multirow{24}{*}{$\begin{array}{l}\text { Rare earth elements in fly ash created during the } \\
\text { coal burning process in certain coal fired power } \\
\text { plants operating in Poland - Upper Silesian } \\
\text { Industrial Region, Danuta Smolka Danielowska, } \\
\text { Faculty of Earth Sciences, University of Silesia, } \\
\text { Poland, } 2010\end{array}$} & 1 & - & $84 \pm 3$ & - & $32 \pm 5$ & $\begin{array}{c}14,6 \\
\pm 1\end{array}$ & $\begin{array}{l}732 \\
\pm 20\end{array}$ & - & $\begin{array}{c}102 \pm \\
2\end{array}$ \\
\hline & 2 & $\begin{array}{c}177 \pm \\
20 \\
\end{array}$ & $52 \pm 2$ & - & $78 \pm 3$ & $\begin{array}{c}5,6 \pm \\
0,5 \\
\end{array}$ & $\begin{array}{l}740 \\
\pm 20 \\
\end{array}$ & - & $\begin{array}{c}102 \pm \\
2 \\
\end{array}$ \\
\hline & 3 & $\begin{array}{c}99 \pm 3 \\
2\end{array}$ & $99 \pm 4$ & - & $79 \pm 2$ & $\begin{array}{l}12,3 \\
\pm 1,5\end{array}$ & $\begin{array}{l}772 \\
\pm 19\end{array}$ & - & $73 \pm 2$ \\
\hline & 4 & $\begin{array}{c}137 \pm \\
38\end{array}$ & $82 \pm 3$ & - & $69 \pm 3$ & $\begin{array}{c}16,5 \\
\pm 1\end{array}$ & $\begin{array}{l}682 \\
\pm 19\end{array}$ & - & $72 \pm 2$ \\
\hline & 5 & $78 \pm 2$ & $\begin{array}{c}135 \pm \\
3 \\
\end{array}$ & - & $33 \pm 3$ & $\begin{array}{c}12 \pm \\
1,1 \\
\end{array}$ & $\begin{array}{l}624 \\
\pm 23 \\
\end{array}$ & - & $84 \pm 3$ \\
\hline & 6 & $\begin{array}{c}56 \pm 1 \\
6\end{array}$ & $\begin{array}{c}134 \pm \\
3\end{array}$ & - & $38 \pm 3$ & $\begin{array}{l}6 \pm \\
1,9 \\
\end{array}$ & $\begin{array}{l}578 \\
\pm 20\end{array}$ & - & $72 \pm 2$ \\
\hline & 7 & $\begin{array}{c}20 \pm \\
8,2 \\
\end{array}$ & $77 \pm 3$ & - & $58 \pm 5$ & $\begin{array}{c}18 \pm \\
1,5\end{array}$ & $\begin{array}{r}389 \\
\pm 15 \\
\end{array}$ & - & $99 \pm 4$ \\
\hline & 8 & - & $34 \pm 2$ & - & $12 \pm 1$ & $\begin{array}{c}18 \pm \\
0,5 \\
\end{array}$ & $\begin{array}{l}377 \\
\pm 16 \\
\end{array}$ & - & $\begin{array}{c}100 \pm \\
2 \\
\end{array}$ \\
\hline & 9 & - & $78 \pm 3$ & - & $16 \pm 2$ & $\begin{array}{c}8,3 \pm \\
2,3 \\
\end{array}$ & $\begin{array}{r}760 \\
\pm 19 \\
\end{array}$ & - & $99 \pm 2$ \\
\hline & 10 & - & $64 \pm 3$ & - & $18 \pm 2$ & $18 \pm 1$ & $\begin{array}{l}720 \\
\pm 17\end{array}$ & - & $\begin{array}{c}111 \pm \\
4\end{array}$ \\
\hline & 11 & $\begin{array}{c}175 \pm \\
23 \\
\end{array}$ & $\begin{array}{c}104 \pm \\
2 \\
\end{array}$ & - & $76 \pm 3$ & $\begin{array}{r}12,4 \\
\pm 1,5 \\
\end{array}$ & $\begin{array}{r}735 \\
\pm 19 \\
\end{array}$ & - & $26 \pm 2$ \\
\hline & 12 & $\begin{array}{c}66 \pm \\
23 \\
\end{array}$ & $\begin{array}{c}118 \pm \\
3 \\
\end{array}$ & - & $49 \pm 4$ & $\begin{array}{c}10 \pm \\
1,8 \\
\end{array}$ & $\begin{array}{l}679 \\
\pm 21 \\
\end{array}$ & - & $25 \pm 2$ \\
\hline & 13 & $\begin{array}{c}72 \pm 1 \\
4\end{array}$ & $\begin{array}{c}105 \pm \\
3 \\
\end{array}$ & - & $33 \pm 4$ & $\begin{array}{c}11 \pm \\
1,3\end{array}$ & $\begin{array}{l}733 \\
\pm 19\end{array}$ & - & $72 \pm 2$ \\
\hline & 14 & $\begin{array}{c}176 \pm \\
12 \\
\end{array}$ & $97 \pm 3$ & - & $77 \pm 5$ & $\begin{array}{r}14,8 \\
\pm 1,4\end{array}$ & $\begin{array}{l}795 \\
\pm 24\end{array}$ & - & $53 \pm 2$ \\
\hline & 15 & $\begin{array}{c}130 \pm \\
22\end{array}$ & $\begin{array}{c}141 \pm \\
4\end{array}$ & - & $76 \pm 2$ & $16 \pm 6$ & $\begin{array}{l}724 \\
\pm 16\end{array}$ & - & $89 \pm 2$ \\
\hline & 16 & $48 \pm 8$ & $\begin{array}{c}115 \pm \\
3\end{array}$ & - & $68 \pm 3$ & $16 \pm 1$ & $\begin{array}{l}788 \\
\pm 17\end{array}$ & - & $99 \pm 3$ \\
\hline & 17 & $\begin{array}{c}178 \pm \\
31\end{array}$ & $99 \pm 3$ & - & $44 \pm 4$ & $\begin{array}{r}12,6 \\
\pm 1,8\end{array}$ & $\begin{array}{l}698 \\
\pm 14\end{array}$ & - & $\begin{array}{c}101 \pm \\
2\end{array}$ \\
\hline & 18 & $\begin{array}{c}177 \pm \\
18 \\
\end{array}$ & $\begin{array}{c}111 \pm \\
3 \\
\end{array}$ & - & $32 \pm 5$ & $\begin{array}{r}10,7 \\
\pm 1,8 \\
\end{array}$ & $\begin{array}{r}786 \\
\pm 17 \\
\end{array}$ & - & $\begin{array}{c}102 \pm \\
2 \\
\end{array}$ \\
\hline & 19 & - & $\begin{array}{c}103 \pm \\
3 \\
\end{array}$ & - & $36 \pm 3$ & $\begin{array}{r}11,5 \\
\pm 1,2 \\
\end{array}$ & $\begin{array}{l}771 \\
\pm 21 \\
\end{array}$ & - & $88 \pm 2$ \\
\hline & 20 & - & $\begin{array}{c}121 \pm \\
3 \\
\end{array}$ & - & $58 \pm 3$ & $\begin{array}{r}17,2 \\
\pm 1,6 \\
\end{array}$ & $\begin{array}{r}790 \\
\pm 19 \\
\end{array}$ & - & $86 \pm 3$ \\
\hline & 21 & $\begin{array}{c}168 \pm \\
21 \\
\end{array}$ & $\begin{array}{c}112 \pm \\
3 \\
\end{array}$ & - & $56 \pm 5$ & $18 \pm 1$ & $\begin{array}{r}766 \\
\pm 18 \\
\end{array}$ & - & $\begin{array}{c}100 \pm \\
2 \\
\end{array}$ \\
\hline & 22 & $\begin{array}{c}99 \pm 1 \\
5\end{array}$ & $\begin{array}{c}107 \pm \\
3\end{array}$ & - & $57 \pm 4$ & $\begin{array}{c}15,9 \\
\pm 1\end{array}$ & $\begin{array}{r}744 \\
\pm 18\end{array}$ & - & $98 \pm 2$ \\
\hline & 23 & $\begin{array}{c}85 \pm 1 \\
7 \\
\end{array}$ & $\begin{array}{c}127 \pm \\
3 \\
\end{array}$ & - & $66 \pm 2$ & $\begin{array}{r}14,7 \\
\pm 1,3 \\
\end{array}$ & $\begin{array}{r}734 \\
\pm 15 \\
\end{array}$ & - & $86 \pm 2$ \\
\hline & 24 & $\begin{array}{c}148 \pm \\
22 \\
\end{array}$ & $\begin{array}{c}119 \pm \\
3 \\
\end{array}$ & - & $39 \pm 5$ & $\begin{array}{r}13,8 \\
\pm 1,2 \\
\end{array}$ & $\begin{array}{r}770 \\
\pm 20 \\
\end{array}$ & - & $81 \pm 3$ \\
\hline
\end{tabular}




\begin{tabular}{|c|c|c|c|c|c|c|c|c|c|}
\hline $\begin{array}{l}\text { Modeling Radionuclides Dispersion and } \\
\text { Deposition Downwind of a Coal-Fired Power } \\
\text { Plant - Dinis M.L., Fiúza A., Góis J., Carvalho } \\
\text { J.M.S., Castro A.C.M., Centre for Natural } \\
\text { Resources and the Environment (CERENA), } \\
\text { Instituto Superior Técnico - IST, Av. Rovisco } \\
\text { Pais, Lisabona, Portugalia }\end{array}$ & 1 & $\begin{array}{c}53,9 \\
7\end{array}$ & $\begin{array}{c}40,1 \\
3\end{array}$ & - & - & - & $\begin{array}{r}901 \\
, 44\end{array}$ & - & - \\
\hline $\begin{array}{l}\text { 4. Conclusions } \\
\text { Concentrations of natural radionuclides in } \\
\text { ash dumps do not lead to a significant } \\
\text { increase in the absorbed dose rate, as } \\
\text { evidenced by measurements with ionizing } \\
\text { radiation flow meters on the heap surfaces } \\
\text { at millimeter distances. } \\
\text { Particular attention must, however, be given }\end{array}$ & & $\begin{array}{l}\text { these } \\
\text { concent } \\
\text { where } n \\
\text { especial } \\
\text { Commis } \\
\text { through } \\
\text { itself to } \\
\text { the conc }\end{array}$ & $\begin{array}{l}\text { ration } \\
\text { nan is } \\
\text { ly sin } \\
\text { ssion } \\
\text { its st } \\
\text { the ri } \\
\text { centrat }\end{array}$ & & $\begin{array}{l}\mathrm{CR} \\
\log \end{array}$ & $\begin{array}{l}\text { (Inte } \\
\text { Pr }\end{array}$ & $\begin{array}{l}\text { ify } \\
\text { spar } \\
\text { h lon } \\
\text { natio } \\
\text { tectio } \\
\text { s, ale } \\
\text { posed } \\
\text { e gas. }\end{array}$ & & \\
\hline
\end{tabular}

\section{References}

[1] Moșteanu D., Ilie F., Study of the a radioactivity of the coal of sărmăşag in the county of Sălaj., Scientific magazine 18/2, Land Forces Academy, June, Sibiu, Romania, 2013.

[2] Moșteanu D., Trefaş L., Research on beta-radioactivity of water samples from Sibiu, The XVIIIth International Conference, Land Forces Academy, June, Sibiu, Romanai, 2013.

[3] Giurgiu L., Bârsan G., Moșteanu D., Web Syndication in Educational Enviroment, Proceedings of 50th International Symposium ELMAR, pp. 10-12, Zadar, Croatia, September, 2008.

[4] Moșteanu D., Mihăila-Lica G., Halmaghi E., Mosteanu R., Revista Academiei Forţelor Terestre, Vol. 1, No. 73, pp 106-113, 2014.

[5] Mosteanu D., Margin C., Bârsan G., Giurgiu L., Buletinul ştiinţific al Academiei Forţelor Terestre, Vol. 2, No. 36, pp. 50-55, 2014.

[6] Moşteanu D., MARGIN C, Florin I., Buletinul ştiinţific al Academiei Forţelor Terestre, Vol. 1, No, 37, pp. 50-55, 2014.

[7] P. Soudek, Š. Valenová, Z. Vavř́íková and T. Vaněk, Journal of Environmental Radioactivity, Vol. 88, pp. 236-250, 2006.

[8] Ionel I., Ungureanu C., Power system and environment, ed. Tehnică, Bucureşti, 1996.

[9] Bungau S., Baldea I., Copolovici L., Revista de Chimie-Bucharest, Vol. 54, No. 3, pp. 213-216, 2003.

[10] Bungau S., Bungau C., Tit D. M., Journal of Environmental Protection and Ecology, Vol. 16, No. 1, pp. 56-62, 2015.

[11] Bungau S., Suciu R., Bumbu A., Cioca G., Tit D. M., Journal of Environmental Protection and Ecology, Vol. 16, No. 3, pp. 980-987, 2015.

[12] Ionescu G., Rada E. C., Cioca L.I. Municipal solid waste sorting and treatment schemes for the maximization of material and energy recovery in a latest EU member, Proc. 10th International Conference on Environmental Legislation, Safety Engineering and Disaster Management, Cluj-Napoca, Romania, Vol. 14, No. 11, pp. 2537-2544, 2015.

[13] Nechifor G., Ajmone-Marsan F., Environmental Science. Processes \& Impacts, Vol. 16, No. 2, pp. 211-219, 2014. 
[14] Popescu D. E., Bungau C., Prada M., Domuta C., Bungau S., Tit D. M., Journal of Environmental Protection and Ecology, Vol. 17, No. 3., pp. 1011-1020, 2016.

[15] Rada E.C., Cioca L.I., Energy Procedia, Vol. 119C, pp. 72-85, 2017.

[16] Rada E.C., Cioca L.I., Ionescu G. Energy recovery from Municipal Solid Waste in EU: proposals to assess the management performance under a circular economy perspective. In MATEC Web of Conferences, Vol. 121, pp. 05006, 2017

[17] Ragazzi M., Maniscalco M., Torretta V., Ferronato N., Rada E.C., Energy Procedia, Vol. 119, pp. 602-614, 2017.

[18] Taschina M., Copolovici D. M., Bungau S., Lupitu A. I., Copolovici L., Iovan C., Farmacia, Vol. 65, No. 5, pp. 709-713, 2017.

[19] Tit D. M., Bungau S., Nistor Cseppento C., Copolovici D. M., Buhas C., Journal of Environmental Protection and Ecology, Vol. 17, No. 4, pp. 1425-1433, 2016.

[20] Copolovici D., Bungau S., Boscencu R., Tit D. M., Copolovici L., Revista de Chimie-Bucharest, Vol. 68, No. 3, pp. 507-509, 2017.

[21] Epure G., Grigoriu N., Moşteanu D. E, Revista Academiei Forţelor Terestre, Vol. 2, No. 78, pp. 226-232, 2015.

[22] Epure G., Grigoriu N., Moşteanu D. E., Buletinul ştiinţific al Academiei Forţelor Terestre, Vol. 1, No. 39, pp. 49-56, 2015.

[23] Gitea D., Vicas S., Gitea M. A., Nemeth S., Tit D. M., Pasca B., Purza L., Iovan C., Revista de Chimie-Bucharest, Vol. 69, No. 2, pp. 305-309, 2017.

[24] Moşteanu D., Miclăuş S., Bârsan G., Reasearch Regarding the Obtaining Volatile Oils from Native Plants in an under Vacuum Microwave System, The 17th European Symposium on Computer Aided Process Engineering - ESCAPE17, Bucuresti, Romania, mai 2007.

[25] Mot C. A., Lupitu A. I., Bungau S., Copolovici D. M., Purza L., Melinte (Frunzulica) C. E., Copolovici L., Revista de Chimie-Bucharest, Vol. 69, No. 5, in press, 2018.

[26] Oprea O. B., Apostol L., Bungau S., Cioca G., Samuel A. D., Badea M., Gaceu L., Revista de Chimie-Bucharest, Vol. 69, No. 1, pp. 70-75, 2018.

[27] Pallag A., Bungau S. G., Tit D.M., Jurca T., Sirbu V., Honiges A., Horhogea C., Revista de Chimie-Bucharest, Vol. 67, No. 3, pp. 530-533, 2016.

[28] Mosteanu, D., Trefas, L., Trefas, A.S., Mosteanu, R., Risk other than attack substances transportation, The 21st International Conference Knowledge Based Organization, Nicolae Balcescu Land Forces Academy, pp. 857-860, Sibiu, Romania, 2015.

[29] Reveica I.M., Radioactivity and radioactive isotope circuit in the environment, Ed. Universităţii din Bucureşti, 1998.

[30] http://www.unscear.org/unscear/en/publications/2008_1.html. 